Symptom reduction and improved function in chronic CRPS type 1 after 12-week integrated, interdisciplinary therapy

\title{
Elomaa, Minna
}

2019-04

Elomaa , M , Hotta , J , Williams , A C D C , Forss , N , Äyräpää , A , Kalso , E \& Harno , H 2019 , ' Symptom reduction and improved function in chronic CRPS type 1 after 12-week integrated, interdisciplinary therapy ', Scandinavian journal of pain , vol. 19 , no. 2 , pp. 257-270 . https://doi.org/10.1515/sjpain-2018-0098

http://hdl.handle.net/10138/314242

https://doi.org/10.1515/sjpain-2018-0098

cc_by_nd

publishedVersion

Downloaded from Helda, University of Helsinki institutional repository.

This is an electronic reprint of the original article.

This reprint may differ from the original in pagination and typographic detail.

Please cite the original version. 


\section{Clinical pain research}

\section{Minna Elomaa ${ }^{a}$, Jaakko Hottaa , Amanda C de C Williams, Nina Forss, Anni Äyräpää, Eija Kalso and Hanno Harno* \\ Symptom reduction and improved function in chronic CRPS type 1 after 12-week integrated, interdisciplinary therapy}

https://doi.org/10.1515/sjpain-2018-0098

Received June 1, 2018; revised October 23, 2018; accepted October 29, 2018; previously published online February 21, 2019

\begin{abstract}
Background and aims: Complex Regional Pain Syndrome (CRPS) often recovers spontaneously within the first year, but when it becomes chronic, available rehabilitative therapies (pharmacological management, physiotherapy, and psychological intervention) have limited effectiveness. This study examined the effect of a 12-week intensive outpatient rehabilitation on pain relief and function in chronic CRPS patients. Rehabilitation program included memantine and morphine treatment (added to patient's prior pain medication) and concurrent psychological and physiotherapeutic intervention. Primary outcome measure was a change in CRPS symptom count and secondary
\end{abstract}

${ }^{a}$ Minna Elomaa and Jaakko Hotta: These authors contributed equally to this work.

*Corresponding author: Hanno Harno, Pain Clinic, Department of Anaesthesiology, Intensive Care and Pain Medicine, Helsinki University Hospital and University of Helsinki, Helsinki, Finland; and Pain Clinic, Department of Clinical Neurosciences, Neurology, Helsinki University Hospital and University of Helsinki, P.O Box 140, 00029 HUS, Helsinki, Finland, Phone: +358504639469 ,

Fax:+358947175641, E-mail: hanna.harno@hus.fi

Minna Elomaa and Eija Kalso: Pain Clinic, Department of Anaesthesiology, Intensive Care and Pain Medicine, Helsinki University Hospital and University of Helsinki, Helsinki, Finland Jaakko Hotta and Nina Forss: Clinical Neurosciences, Neurology, Helsinki University Hospital and University of Helsinki, Helsinki, Finland; and Department of Neuroscience and Biomedical Engineering, Aalto University, Espoo, Finland

Amanda C de C Williams: Research Department of Clinical, Educational and Health Psychology, University College London, London, UK

Anni Äyräpää: Pain Clinic, Department of Anaesthesiology, Intensive Care and Pain Medicine, Helsinki University Hospital and University of Helsinki, Helsinki, Finland; and Internal Medicine and Rehabilitation, Physiotherapy, Helsinki University Hospital and University of Helsinki, Helsinki, Finland outcomes were motor performance, psychological factors, pain intensity, and quality of life.

Methods: Ten patients with chronic upper limb CRPS I (median 2.9 years, range 8 months to 12 years) were recruited to the study and were assessed before and after the intervention. Hand motor function of the patients was evaluated by an independent physiotherapist. There were standardized questionnaires for depression, pain anxiety, pain acceptance, quality of life, and CRPS symptom count. In addition, psychological factors were evaluated by a semi-structured interview. Severity of experienced pain was rated at movement and at rest. In addition, a video experiment of a hand action observation was conducted pre- and post-intervention to study possible change in neuronal maladaptation. Intervention consisted of pharmacological, psychological and physiotherapeutic treatment. First, $10 \mathrm{mg}$ daily morphine was started and increased gradually to $30 \mathrm{mg}$ daily, if tolerated. After $30 \mathrm{mg} /$ day or tolerated dose of morphine was achieved, $5 \mathrm{mg}$ daily memantine was started and increased gradually to $40 \mathrm{mg}$, if tolerated. Psychological intervention consisted of weekly group sessions, using cognitive and behavioral methods (relaxation, behavioral activation, and exposure) and acceptance and commitment therapy (ACT) and daily home practice. Physiotherapeutic intervention consisted of graded motor imagery and physiotherapy exercises with weekly group sessions and/or individual guidance by the physiotherapist, and individual exercise of the affected upper limb.

Results: Multimodal intensive intervention resulted in significant decrease in CRPS symptom count. The effect was strongest in motor and trophic symptoms (19\% decrease after intervention) and in sensory symptoms (18\% decrease). Additionally, improvement was seen in some, but not all, secondary outcomes (movement pain, motor symptoms, change in perceptions during video experiment of hand actions, and summary index with motor functioning, pain, and psychological factors). There were no dropouts. 
Conclusions: Intensive 12-week multimodal intervention reduced some CRPS symptoms but was not sufficient to alter patients' rest pain, distress, or quality of life.

Implications: These results support the efficacy of an interdisciplinary rehabilitation program for pain and function in chronic CRPS patients. After intervention, some CRPS symptoms reduced and function improved, but distress and quality of life were unchanged. This may be due to the relatively short duration of this program; to delayed effects; to particular cognitive problems of CPRS patients; and/or to low distress levels at baseline that make statistically significant reduction less likely.

Keywords: CRPS; multidisciplinary; interdisciplinary; intervention; pain; symptom.

\section{Introduction}

Complex Regional Pain Syndrome (CRPS) is a disabling pain condition that may develop after extremity trauma or surgery. CRPS type 1 is defined as having no peripheral nerve lesion, while CRPS type 2 requires it. The pain in CRPS is regional and accompanied by many symptoms such as hyperalgesia, allodynia, swelling, abnormal sweating, movement restriction, weakness, tremor, dystonic postures, trophic changes in skin, nails, or hair, difference in temperature, or changes in color of the CRPS limb [1]. The outcome and prognosis of CRPS are highly variable. Although symptoms often improve significantly over the first year, only about 5\% resolve [2] and many people have persistent CRPS with severe functional impairment and decreased quality of life [3, 4].

An interdisciplinary treatment is widely accepted for CRPS patient rehabilitation, but the content varies [5-7]. A major obstacle in the rehabilitation of CRPS is that motor actions aggravate limb pain [8], leading to avoidance of movement, disuse of the affected extremity, and attention directed towards signals of pain exacerbation $[5,9,10]$. This may interact with mechanisms that maintain CRPS. Graded Motor Imagery (GMI) is applied to train the brain's motor circuitry without actual movement using sequential exercises including laterality training, imagined hand movements, and mirror therapy. GMI may reduce pain and enhance limb function better than conservative physiotherapy [11], but early reports of effectiveness of multimodal physiotherapy to treat CRPS have not been consistently replicated [12].

Psychological interventions can enhance coping with chronic pain and ability to self-manage pain.
Psychological treatments for CRPS usually include cognitive behavioral therapy (CBT) consisting of relaxation training, exposure therapy, and goal-directed behavioral activation. These aim to reduce fear of movement, to modify unhelpful beliefs and catastrophic thinking about CRPS, [13] and to support the patient in working towards valued goals. Acceptance and commitment therapy (ACT) and mindfulness-based interventions for chronic pain patients emphasize acceptance, personal values and goals, reflected in thoughts and actions [14].

In this study, we conducted a structured rehabilitation program for chronic upper limb CRPS patients to study the effectiveness of interdisciplinary treatment with an integrated combination of pharmacological, psychological and physiotherapeutic interventions. The rehabilitation included self-practice supported by sessions with a physiotherapist (individual therapy and GMI) and a psychologist (CBT and ACT) emphasizing exposure to previously avoided situations, practiced first in imagined and then in real situations. The pharmacological intervention aimed to reduce pain to facilitate the psychological and physical rehabilitation [15]. Given that the program aimed at rehabilitation from a difficult-to-treat pain condition, outcomes were broad.

\section{Materials and methods}

\subsection{Patients}

The patients were recruited mainly from the Pain Clinic of the Helsinki University Hospital and from two other Hospitals at the Uusimaa district as part of a larger CRPS study which included MRI and video experiments [16-19]. Inclusion criteria were age from 18 to 65, CRPS type 1 (Budapest criteria [1]) in the upper limb for at least 6 months, and during the past week the maximum intensity of pain more than four on a 11-point numeric rating scale (NRS 0-10, $0=$ no pain, $10=$ extreme pain). Exclusion criteria were major psychiatric or neurological diseases and alcohol or drug abuse by self-report. Additionally, no previous use of memantine, which was part of the study medication, was allowed and mild opioids were tapered down before study medication.

\subsection{Study protocol}

The study was a 12-week open label interdisciplinary, integrated treatment with pharmacological, 
physiotherapeutic, and psychological interventions. It was conducted in two parts with six patients in 2011 (patients p11-p16) and four patients in 2014 (p21-p24). Treatment started with a 2-week titration of morphine and memantine that were added to the pre-existing neuropathic pain medication. Thereafter, psychological and physiotherapy interventions started and lasted for 9 and 7 weeks, respectively. Physiotherapy and psychological intervention were conducted in a synchronized manner to encourage the patients to practice new skills to improve functioning. With tapering off of study medication, the total study period was 12 weeks (see Fig. 1).

A neurologist $(\mathrm{HH})$ conducted a thorough sensory and motor examination with multiple questionnaires including CRPS symptom count. An independent physiotherapist performed a detailed motor examination. A psychologist (ME) conducted a semistructured interview and patients completed several standardized questionnaires on psychological factors. In addition, a video experiment with hand actions was conducted before and after intervention $(\mathrm{JH})$.

\subsubsection{Pharmacological intervention}

The pharmacological intervention aimed to reduce pain before starting the physiotherapeutic and psychological interventions. The choice of medication was based on a previous, double-blind randomized placebo-controlled study on CRPS patients with morphine and memantine (NMDA-receptor antagonist) that showed pain reduction with concomitant changes in brain areas associated with pain [15]. The pre-study neuropathic pain medication was continued to reach best possible pain control.

First, the patients started with oral morphine $10 \mathrm{mg}$ daily and increased the dose every 3 days to $10 \mathrm{mg}$ three times a day, if tolerated. After a week, oral memantine $5 \mathrm{mg}$ daily was added and the dose was every 3 days increased up to $40 \mathrm{mg}$ per day, if tolerated. These medications were used in addition to regular neuropathic pain

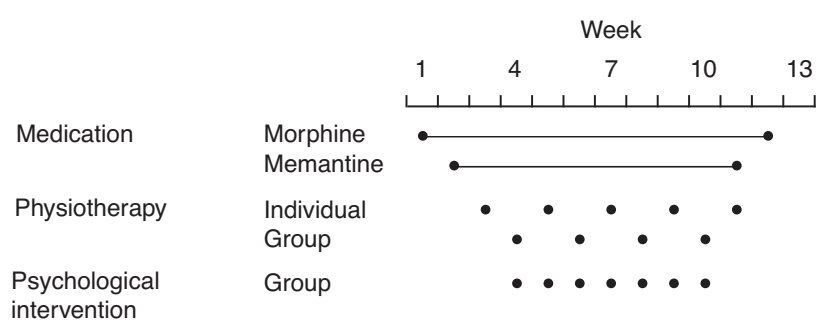

Fig. 1: Study plan of CRPS intergraded intervention. medications (gabapentinoids in seven patients, tricyclics in three, serotonin-norepinephrine reuptake inhibitors in three, and simple analgesics in three). Tramadol or acetaminophen with codeine were discontinued before oral morphine intake started (six patients). The patients continued the study medication throughout, if tolerated, and used no additional analgesics. To monitor side-effects, patients completed a checklist-diary that provided examples (constipation, nausea, vomiting, dizziness, headache, drowsiness, visual symptoms, dyspnea) from which patient could endorse as many as required. Each symptom was rated on NRS-11 $(0=$ no symptom, $10=$ worst possible symptom) at least once a week.

\subsubsection{Physiotherapy}

Physiotherapy started with laterality exercises based on Graded Motor Imagery (GMI) [20]. The physiotherapist met the patient once a week in alternating individual and group sessions. Exercises were added gradually and modified individually according to function and pain of the affected upper limb; some exercises were computerbased. Patients were encouraged to use both hands in daily activities. The detailed physiotherapy intervention is presented in Table 1.

GMI is a three-step training program that starts with laterality training in which patients judge whether the limb presented in a flash card and/or in a special computer program is the right or left-sided limb. Second, imagery exercises involve imagining the limb in a certain posture or a movement presented in a flash card and/or in a computer program (online, www.noigroup.com/recognize). Third, mirror therapy progresses from exercises where the patient follows mainly the reflection of the healthy upper limb actions in the mirror while the CRPS hand rests inside a mirror box. A more intense exercise includes actual actions of the CRPS hand [20].

Training of the upper limb included an individually planned progressive training program for each patient with the physiotherapist. Upper limb exercises, such as grip, dexterity, and selective movements of one finger, were performed every week. Desensitization training consisted of two-point discrimination using cocktail sticks and dexterity exercises with small objects such as beads of different sizes. Physiotherapy included stretching and strength training with hand weights and exercise bands. General neck-shoulder, upper body-focused exercises, balance and postural control, body perception, and image exercises were also practiced in group sessions. 
Table 1: The content of physiotherapy intervention.

\begin{tabular}{ll}
\hline Week & Techniques \\
\hline 1. Introduction to GMI & Laterality exercise, individual guidance \\
2. Laterality exercises (group) & Laterality exercises, guided physiotherapy \\
3. Mental imagery exposure & Computer assessed mental imagery and use of flashcards with hand posture pictures \\
4. Mental imagery (group) & Guided physiotherapy, exercises for the affected limb and body posture \\
5. Start of mirror therapy & Mirror therapy \\
6. Mirror therapy and previous exercises (group) & Guided physiotherapy, exercises for the affected limb and body posture, mirror therapy \\
7. Mirror therapy & Mirror therapy, monitoring of the progress \\
8. Exercise (group) & Mirror therapy, exercises of the affected limb, upper body and posture \\
9. Control & Practice summary and a plan for an individual exercise program for the affected limb for \\
& the future \\
\hline
\end{tabular}

$\mathrm{GMI}=$ graded motor imagery .

\subsubsection{Psychological intervention}

The psychological intervention was conducted in seven weekly 90-min group sessions, using cognitive and behavioral methods (relaxation, behavioral activation, and exposure) and acceptance and commitment therapy (ACT) $[14,21]$ (Table 2). Exposure to previously avoided situations was of importance, first practice in imagined and then in real situations. A variety of ACT techniques was taught; body scan, awareness of breathing, attention management exercises, and mindfulness in activities of everyday living.

Pain management was discussed, aiming at perspective change from symptom reduction to symptom control, increased functioning, and quality of life. When new pain management strategies were introduced, patients were instructed to add them to the previous ones. We encouraged patients to combine exercises to fit individual needs. Patients were instructed to practice 20-30 min daily and filled in home practice logs of daily

Table 2: The content of psychological intervention.

\begin{tabular}{|c|c|c|}
\hline Week & Aims & Techniques \\
\hline 1. Introduction to ACT & $\begin{array}{l}\text { Introduction to the treatment. Discussion of pain avoidance and its' } \\
\text { consequences, shifting perspective from pain and symptom reduction to } \\
\text { valued living }\end{array}$ & $\begin{array}{l}\text { Brief relaxation with focus } \\
\text { on breathing }\end{array}$ \\
\hline $\begin{array}{l}\text { 2. Basic attention } \\
\text { skills and mindfulness }\end{array}$ & To increase body awareness and non-judgmental observing skills & $\begin{array}{l}\text { Internal and external } \\
\text { attention, body awareness } \\
\text { and breathing }\end{array}$ \\
\hline $\begin{array}{l}\text { 3. Use of imagery } \\
\text { exposure }\end{array}$ & $\begin{array}{l}\text { To guide the participants to create vivid imagery of personally valued and } \\
\text { pleasant activity. Participant practice observing all kind of sensations, } \\
\text { both distressing, painful and pleasant associated with activity. Explaining } \\
\text { the importance of fully exposing oneself to all aspects of the experience }\end{array}$ & $\begin{array}{l}\text { Creating vivid images for } \\
\text { imagery exercises and } \\
\text { sensate focus }\end{array}$ \\
\hline $\begin{array}{l}\text { 4. Exposure and } \\
\text { mindfulness in } A D L\end{array}$ & $\begin{array}{l}\text { To increase the use of the CRPS hand in daily activities. Mindfulness as } \\
\text { exposure exercise in ADL }\end{array}$ & $\begin{array}{l}\text { Mindfulness and exposure } \\
\text { in daily activities }\end{array}$ \\
\hline $\begin{array}{l}\text { 5. Cognitive de-fusion } \\
\text { and values based } \\
\text { action }\end{array}$ & $\begin{array}{l}\text { To clarify and identify personal values and increase flexibility in behavioral } \\
\text { repertoire. Discussion and encouragement to increase previously avoided } \\
\text { activities. To explore the fusion of emotions, automatic cognitions and } \\
\text { learned responses and how they have led to limited behavioral responses }\end{array}$ & $\begin{array}{l}\text { Defusing catastrophizing } \\
\text { and vicious circles }\end{array}$ \\
\hline $\begin{array}{l}\text { 6. Values based action } \\
\text { and acceptance }\end{array}$ & To continue experimental learning & $\begin{array}{l}\text { Discussion of dealing with } \\
\text { pain and its chronic nature }\end{array}$ \\
\hline $\begin{array}{l}\text { 7. Coping strategies } \\
\text { setting goals }\end{array}$ & To help participants to integrate new skills in their life & $\begin{array}{l}\text { Discussion of all sessions } \\
\text { and use of skills }\end{array}$ \\
\hline
\end{tabular}

$A C T=$ acceptance and commitment therapy; $A D L=$ activities of daily living. 
exercise types and amounts, with comments on their experience.

\subsection{Outcome measures}

The primary outcome was change in the CRPS symptom count, the sum of the patient-reported CRPS symptom descriptors [22]. The secondary outcomes were motor (weakness, movement restriction, dexterity) and psychological variables, pain, health-related quality of life, and changes in perceptions during observation of videos of hand motor actions. Additionally, a summary index of the study outcomes (motor functioning, pain, and psychological wellbeing) was performed.

\subsubsection{Primary outcome}

\subsubsection{CRPS symptom count}

For CRPS symptom count, patients reported on a questionnaire with dichotomous scale $(0=$ no, $1=$ yes $)$ the presence of the following symptoms of the affected limb: hyperalgesia, allodynia, swelling, abnormal sweating, movement restriction, weakness, tremor, dystonic postures, trophic changes in skin, nails, or hair, differences in temperature or color, or changes in color [1].

\subsubsection{Secondary outcomes}

\subsubsection{Motor variables}

Assessment of motor functions were performed by an independent physiotherapist and included measures of hand strength, active ranges of motion (AROMs), and dexterity from healthy and affected sides. The hand grip strength was measured with Jamar dynamometer (position II), and tip and key pinch with a Pinch gauge. A goniometer was used to measure AROMs in extension of fingers II-V, radial abduction of the thumb, dorsal and volar flexion of the wrist, flexion and extension of the elbow, and flexion of the shoulder. In addition, flexion of fingers II-V was recorded by distance $(\mathrm{cm})$ between fingertips and palm in maximum flexion and, similarly, for the thumb opposition, between the tip of the finger $\mathrm{V}$ and the thumb. To assess hand dexterity, the Nine Hole Peg Test (9-HPT) was applied [23]. The subjective level of motor disability was assessed with Disabilities of Arm, Shoulder, and Hand (DASH) questionnaire [24].

\subsubsection{Psychological variables}

The patients received an information sheet describing the content and aims of the psychological treatment. The pretreatment assessment with a semi-structured interview was less than 1 month before starting the intervention and post-treatment assessment less than 1 month after it. In the interview patients were asked about their attitude towards pain, thoughts and emotions, their experience about participation in the group, and which exercises they thought best suited for their needs, pain acceptance, and coping.

The patients completed the following psychological questionnaires: Beck Depression Inventory (BDI-II), a self-administered questionnaire with 21 items measuring depressive symptoms of varying severity, scored $0-3$, with higher scores representing worse symptoms [25]; the Chronic Pain Acceptance Questionnaire (CPAQ), a scale of acceptance of pain from a functional contextual point of view, with 20 items scores on a seven-point scale ( $0=$ never true, $6=$ always true). The Finnish version of the CPAQ has shown good internal consistency (mean alpha $=0.86)$ [26]. The short version of the Pain Anxiety Symptoms Scale (PASS-20), a 20-item scale of pain-related anxiety scores on a six-point scale $(0=$ never, $5=$ always $)$ was also completed. Higher scores mean more pain-related cognitive and physical anxiety, fear, and avoidance. The PASS-20 has demonstrated good internal consistency (mean alpha $=0.81$ ) and has strong correlations (mean $\mathrm{r}=0.95)$ with the original subscales [27].

\subsubsection{Pain}

The pain scale included rating of the maximum and minimum pain (NRS 0-10) during the week before and after the intervention at movement and rest.

\subsubsection{Quality of life}

We used the 15D questionnaire to study health-related quality of life [28]. It includes 15 dimensions that assess different aspects of health functions with five ordinal levels for each dimension in which higher points reflect worse quality of life. Test-retest reliability coefficients of the 15D are $92-100 \%$ depending on the dimension. 15D has recently been validated as a health-related quality of life measure in chronic pain [29].

\subsubsection{Responses to visual sensorimotor actions}

In this experiment, patients first rated pain intensity (NRS-11) while (1) keeping hands still (baseline), and then 
in motor actions of (2) making a fist gently (FIST) and thereafter (3) squeezing given objects with maximum force (SQUEEZE). Then patients observed six first-person-view videos of hand actions from a 13.3-inch laptop display in a quiet environment. In the first two videos the hand was in an upright stationary position (STATIONARY), in the next two making a gentle fist (FIST), and in the last two squeezing objects (SQUEEZE). The first video of each condition always presented a hand corresponding to the patient's healthy hand, and the second corresponding to the patient's affected hand. Each video lasted $32.6 \mathrm{~s}$ and consisted of eight $3.2 \mathrm{~s}$ clips with $1 \mathrm{~s}$ inter-clip intervals; each clip presented the same action but from a slightly different view of the hand. Videos of the left hand were vertically mirrored versions of the right hand-videos. Patients were advised to keep their own hands still while observing the videos.

After each video, the patients marked on a questionnaire (1) the laterality of the presented hand, and then rated on NRS-11 their (2) evaluation of applied force in observed hand actions ( $0=$ no force, $10=$ maximum force), (3) experienced valence $(0=$ very pleasant, $5=$ neutral, $10=$ very unpleasant), and (4) pain level during videos.

Part of the pre-intervention data of the video experiment has already been published elsewhere [16] with a more detailed description of the experimental protocol.

\subsection{Statistical analysis}

The effect of the intervention and the difference between the hands were tested with the paired-sample Wilcoxon signedrank test. In order to study the overall effect of the intervention on motor functions, the normalized motor assessment data for the affected hand were subjected to mixed design ANOVA. Each motor parameter was further analyzed with paired-sample Wilcoxon signed-rank test to study the effects in more detail. To reduce the number of comparisons, only those motor variables that showed significant difference between the affected and healthy limbs at baseline were included in all analyses of intervention effects.

To summarize effects of the intervention, we combined data from the motor tests, pain ratings, and psychological questionnaires into a single subject-wise index. First, change in each was transformed to a categorical variable of 1,0 or -1 , representing improvement, no change, or deterioration respectively. Then the subject-wise index was calculated as the mean of these categorical variables. The indices were tested in the group statistics against 0 with a one-sample Wilcoxon signed-rank test.

We used repeated measures ANOVA to study the effects of the intervention on data from video experiments. Statistically significant effects were analyzed with simple effects tests or planned contrasts. Pre-post changes in pain were tested with paired-sample Wilcoxon signedrank test. For video experiments, the analyses used the data from nine patients as one did not attend the postintervention video experiment (p13).

\section{Results}

\subsection{Patients}

Ten upper limb CRPS type 1 patients (all female, median age 49, range 43-57) attended the study. Demographic and clinical data are presented in Table 3.

Table 3: Demographic and clinical data of the patients.

\begin{tabular}{|c|c|c|c|c|c|c|c|}
\hline Patients & Age & Duration of CRPS & $\begin{array}{l}\text { Hand } \\
\text { affected }\end{array}$ & Handedness & $\begin{array}{l}\text { Oedema/ } \\
\text { color change }\end{array}$ & $\begin{array}{l}\text { Sensory, motor and } \\
\text { trophic symptoms }\end{array}$ & ENMG \\
\hline p11 & 45 & 2 years 4 months & $\mathrm{R}$ & $\mathrm{R}$ & $-1+$ & $\mathrm{A}, \mathrm{D}, \mathrm{H}, \mathrm{T}, \mathrm{M}$ & $\mathrm{N}$ \\
\hline p12 & 43 & 8 years & $R$ & $\mathrm{R}$ & $-1+$ & $A, M$ & $\mathrm{~N}$ \\
\hline p13 & 56 & 12 years & $R$ & $R$ & $-1+$ & $\mathrm{A}, \mathrm{T}, \mathrm{M}$ & $\mathrm{N}$ \\
\hline p14 & 44 & 8 years & $\mathrm{R}$ & $\mathrm{R}$ & $-1-$ & $\mathrm{H}, \mathrm{M}$ & $\mathrm{N}$ \\
\hline p15 & 50 & 2 years 1 months & $\mathrm{R}$ & $\mathrm{R}$ & $+/+$ & $\mathrm{A}, \mathrm{H}, \mathrm{M}$ & $\mathrm{N}$ \\
\hline p16 & 49 & 3 years 5 months & $\mathrm{R}$ & $\mathrm{R}$ & $+/-$ & $\mathrm{A}, \mathrm{M}$ & $\mathrm{N}$ \\
\hline p21 & 49 & 8 months & $\mathrm{R}$ & $R$ & $+1-$ & $A, D, H, M$ & $\mathrm{Fa}$ \\
\hline p22 & 55 & 11 years 2 months & L & L & $-1+$ & $A, D, M$ & $\mathrm{~N}$ \\
\hline p23 & 49 & 2 years 5 months & $\mathrm{R}$ & L & $-1-$ & $\mathrm{A}, \mathrm{H}, \mathrm{M}$ & $\mathrm{N}$ \\
\hline p24 & 57 & 1 year 1 month & L & $R$ & $-1-$ & $\mathrm{A}, \mathrm{T}, \mathrm{M}$ & $\mathrm{Fb}$ \\
\hline
\end{tabular}

$\mathrm{L}=$ left; $\mathrm{R}=$ right; $\mathrm{A}=$ allodynia; $\mathrm{D}=$ dysesthesia; $\mathrm{H}$ = hypersensitivity; $\mathrm{M}$ = motor changes; $\mathrm{T}$ = trophic changes;

ENMG = electroneuromyography; $\mathrm{Fa}=$ minor median nerve lesion. Underwent canalis carpi operation twice, but the same finding after the operation. $\mathrm{Fb}=$ minor partial nervus radialis lesion after left I CMC resection arthroplasty operation; $\mathrm{N}=$ normal. 


\subsection{Pharmacological intervention}

None of the patients were able to complete the pharmacological intervention quite as planned. In all patients, the dose or duration of study medication had to be diminished due to side-effects. Figure 2 illustrates the study medication usage (morphine and memantine). Morphine daily median dosage was $23.3 \mathrm{mg}$ (range 0.0-28.5), and memantine daily median dosage $11.9 \mathrm{mg}$ (range 5.0-23.9) (Fig. 2).

Most common adverse events were dizziness (78\%), sleepiness $(78 \%)$, constipation $(74 \%)$, nausea $(61 \%)$, headache $(61 \%)$ and visual problems (57\%). Overall, patients reported 9.5 \pm 3.3 (mean \pm SD; range 6-15) different adverse events with a mean severity of $3.3 \pm 1.3$ (1-9; NRS-11). None of the patients used morphine or memantine before the study started.

\subsection{CRPS symptom count}

The self-reported CRPS symptom count reduced significantly in post-intervention compared with pre-intervention (Fig. 3A and Table 4). The effect was strongest in the motor/trophic (mean relative reduction $-19 \%$ ), and sensory symptoms $(-18 \%),-14 \%$ in vasomotor symptoms, and $-7 \%$ in the sweating/oedema symptoms (Fig. 3B) [1].

The intervention summary effect (a combination of parameters of motor functioning, psychological

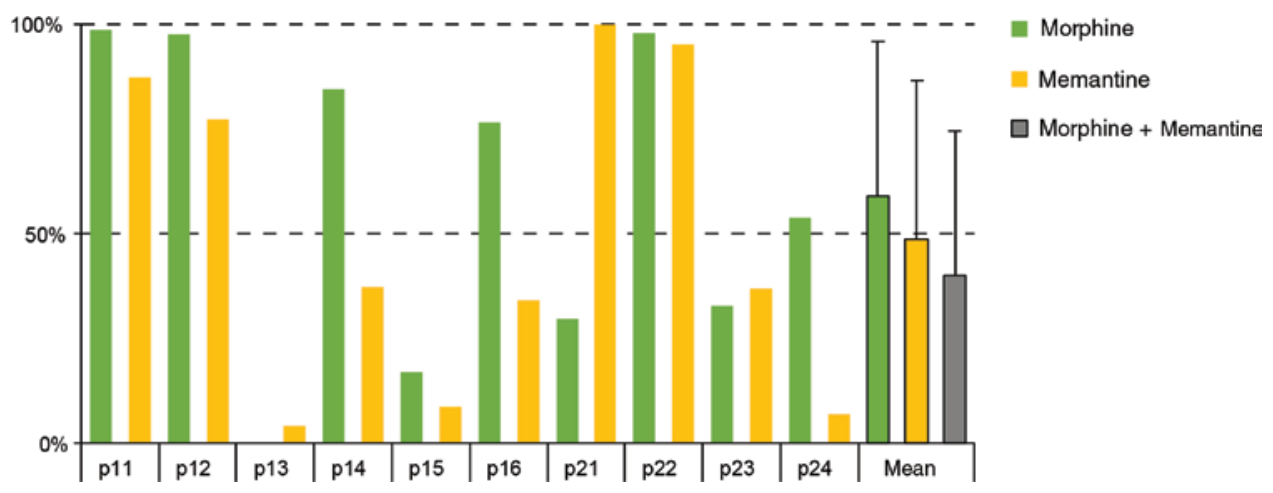

Fig. 2: Percentage of days on scheduled study medication.

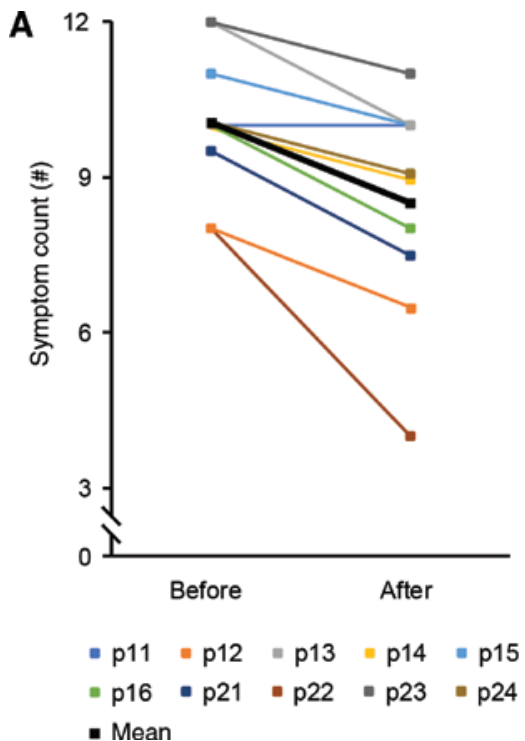

B<smiles>[Z]C(C)(C)C([3H])([3H])[3H]</smiles>

Sweating/Oedema

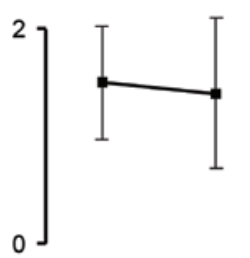

Motor/Trophic<smiles>CC(C)(C)C(C)(C)CS</smiles>

Vasomotor

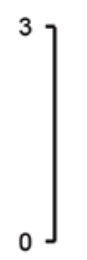<smiles>[3H]C([3H])([3H])[3H]</smiles>

Fig. 3: CRPS symptom count before and after intervention. (A) The CRPS symptom count (maximum =12) before and after the intervention for individual patients (colored thin lines; p01-p24) and mean. (B) The mean \pm SD of the CRPS symptom count displayed in four symptom categories before and after intervention. 
questionnaires, and pain-ratings) showed significant improvement after the intervention (Fig. 4).

\subsection{Motor variables}

Before the intervention, the affected limb was significantly worse than the healthy hand in all hand motor measures: AROMs of thumb radial abduction, wrist extension-flexion movements, shoulder flexion, and 9-HPT (Table 5). The DASH questionnaire showed significant upper-limb disability in patients when compared with normative data [30] (Table 4).
Thumb radial abduction AROM and tip pinch strength showed statistically significant improvement after intervention (Table 5).

\subsection{Psychological variables}

At baseline, before the intervention, all patients showed mild depressive symptoms (range 9-14 points) in BDI and mild anxiety in PASS-20 (mean 45.1, SD 9.7).

In post-intervention, BDI-II, CPAQ, and PASS-20 showed reduction in depressive symptoms, anxiety, and pain-related fear, but did not reach statistical

Table 4: Pre- and postintervention change in outcomes according to study questionnaires.

\begin{tabular}{|c|c|c|c|}
\hline \multirow[t]{2}{*}{ Questionnaire } & \multirow{2}{*}{$\frac{\text { Pre-intervention }}{\text { Mean } \pm \text { SD }}$} & \multirow{2}{*}{$\frac{\text { Post-intervention }}{\text { Mean } \pm \text { SD }}$} & \multirow{2}{*}{$\begin{array}{c}p \text {-Value } \\
\text { Wilcoxon signed-rank tes }\end{array}$} \\
\hline & & & \\
\hline CRPS symptom questionnaire & $10.1 \pm 1.4$ & $8.5 \pm 2.1$ & 0.007 \\
\hline \multicolumn{4}{|l|}{ Symptom count $[\#]$} \\
\hline \multicolumn{4}{|l|}{ Pain questionnaire [NRS-11] ${ }^{\mathrm{a}}$} \\
\hline Maximum during movement & $8.0 \pm 1.6$ & $7.1 \pm 1.7$ & 0.024 \\
\hline Maximum at rest & $6.7 \pm 1.8$ & $6.1 \pm 2.5$ & 0.396 \\
\hline Minimum at rest & $3.5 \pm 2.1$ & $3.4 \pm 2.0$ & 0.851 \\
\hline DASH & $60.0 \pm 13.5$ & $52.0 \pm 16.3$ & 0.093 \\
\hline BDI-II & $16.0 \pm 7.7$ & $14.1 \pm 6.9$ & 0.201 \\
\hline $\mathrm{CPAQ}^{\mathrm{b}}$ & $60.5 \pm 18.5$ & $62.4 \pm 19.3$ & 0.506 \\
\hline PASS-20 & $43.1 \pm 17.4$ & $39.3 \pm 13.9$ & 0.284 \\
\hline $15-\mathrm{D}$ & $0.74 \pm 0.1$ & $0.73 \pm 0.1$ & 0.405 \\
\hline
\end{tabular}

$15-\mathrm{D}=$ health-related quality of life; $\mathrm{BDI}-\mathrm{II}=$ Beck depression inventory; $\mathrm{CPAQ}=$ chronic pain acceptance questionnaire; $\mathrm{DASH}=$ disabilities of arm, shoulder and hand $(n=9)$; PASS-20 = pain anxiety symptoms scale.

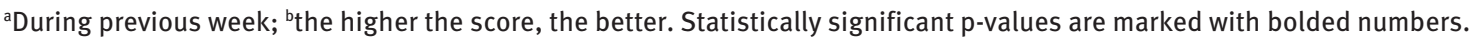
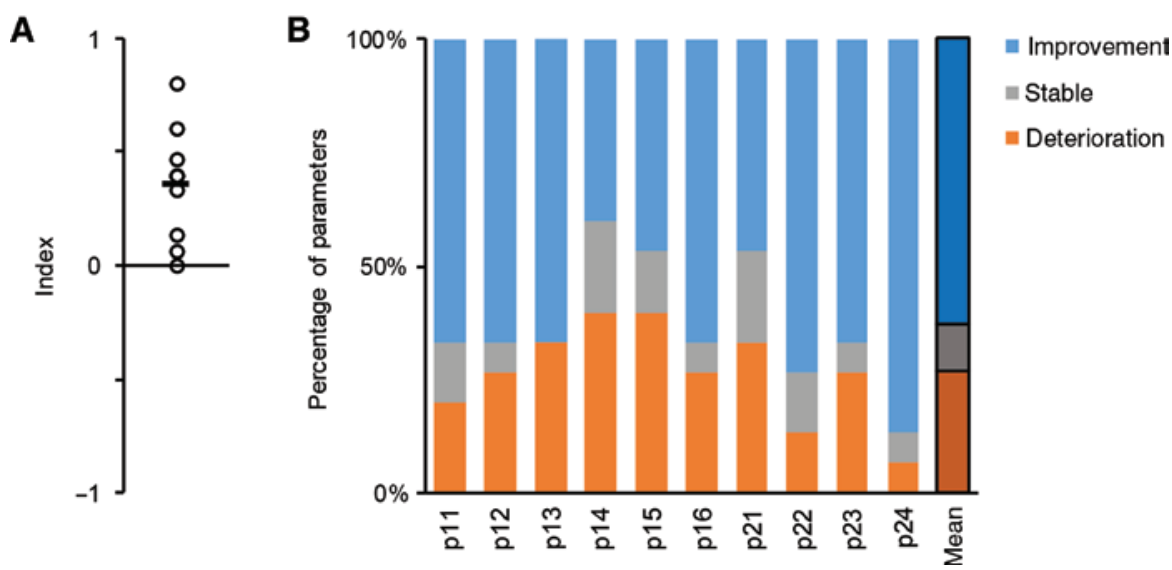

Fig. 4: Intervention summary effect (B) and index for direction of change in symptom parameters (A). The bars represent each patient (B). The index (A) consists of motor functioning, psychological questionnaires, and pain-ratings plotted for each subject (circles) and group mean (black line). 
Table 5: Pre- and postintervention outcome of motor assessments.

\begin{tabular}{|c|c|c|c|c|c|}
\hline \multirow[t]{2}{*}{ Motor assessment } & \multicolumn{3}{|r|}{ Pre-intervent ion } & \multicolumn{2}{|r|}{ Post-intervention } \\
\hline & $\begin{array}{r}\text { Affected hand } \\
\text { median (IQR) }\end{array}$ & $\begin{array}{l}\text { Healthy hand } \\
\text { median (IQR) }\end{array}$ & $\begin{array}{r}p \text {-Value } \\
\text { Wilcoxon signed- } \\
\text { rank test }\end{array}$ & $\begin{array}{r}\text { Affected hand } \\
\text { median (IQR) }\end{array}$ & $\begin{array}{r}p \text {-Value } \\
\text { Wilcoxon signed- } \\
\text { rank test }\end{array}$ \\
\hline \multicolumn{6}{|l|}{ Strength (kg) } \\
\hline Hand grip & $11.0(4.5-20.0)$ & $22.0(20.5-24.8)$ & 0.021 & $14.0(10.0-18.0)$ & 0.858 \\
\hline Tip pinch & $2.6(1.3-3.4)$ & $4.3(3.6-4.7)$ & 0.011 & $3.3(2.0-4.1)$ & 0.042 \\
\hline Key pinch & $3.3(1.3-6.8)$ & $6.8(6.1-7.2)$ & 0.025 & $5.9(3.0-6.8)$ & 0.241 \\
\hline \multicolumn{6}{|l|}{ AROM $\left[^{\circ}\right]$} \\
\hline $\begin{array}{l}\text { Thumb radial } \\
\text { abduction }\end{array}$ & $50(43-58)$ & $70(60-78)$ & 0.008 & $55(49-60)$ & 0.021 \\
\hline Wrist volar flexion & $58(50-65)$ & $80(74-80)$ & 0.008 & $66(56-80)$ & 0.086 \\
\hline Wrist dorsal flexion & $48(33-55)$ & $70(60-79)$ & 0.012 & $53(35-60)$ & 0.463 \\
\hline Shoulder flexion & $143(106-173)$ & $180(170-180)$ & 0.018 & $140(114-180)$ & 0.553 \\
\hline 9HPT [s] $]^{\mathrm{a}}$ & $20.6(19.3-24.7)$ & $19.1(17.4-21.8)$ & 0.032 & $21.1(18.3-22.1)$ & 0.444 \\
\hline
\end{tabular}

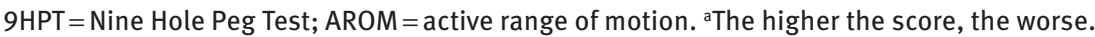

Pre-intervention statistical comparison is between affected and healthy hand. In post-intervention, the affected hand is statistically compared pre-vs. post-intervention. Statistically significant p-values are marked with bolded numbers.

significance (Table 4). However, in post-intervention interview, the patients reported finding psychological intervention in combination with physiotherapy useful. According to the home practice logs, the patients practiced $66 \pm 38$ times including at least two strategies from ACT, CBT, mindfulness, or relaxation. Although most of the participants were familiar with most of the exercises, the intervention intensified their practice and knowledge of CRPS with new ways to manage symptoms. They reported that they could now use the CRPS hand more in activities of daily living ( $n=4)$ and could cope and manage pain better in a variety of situations $(n=5)$. All participants found peer support important, motivating, and beneficial for mood.

Four out of ten patients reported that they had more energy to start and complete activities. Feeling more active was associated with increased functionality of the CRPS hand. Many patients reported that they had started new activities such as gardening or household activities despite pain. Although a small positive change in psychological variables was observed, it did not reach significance in any of the questionnaires (Table 4).

\subsubsection{Present-focused awareness and pain acceptance}

Before intervention, the patients reported having negative feelings about pain. Half of the patients reported clear difficulty in accepting pain. Mindfulness exercises aimed to increase body awareness and non-judgmental observing skills. After the intervention, the participants reported that they had more psychological flexibility and patience with their thoughts and emotions. Although pain evoked negative feelings, present-focused awareness helped patients to "play time" with their reactions. Mindfulness was easier with enjoyable activities. After the intervention five out of ten patients reported that they had learned to accept the presence of pain. They reported increased coping with their pain condition with help of the peer support and a more relaxed attitude on oneself.

Relaxation was mostly used within or combined with other exercises. Six out of 10 patients reported that they benefitted from using relaxation alone or in combination with exercises to calm down when in severe pain.

\subsection{Pain and quality of life}

Intervention significantly reduced the movement pain ( $8.0 \pm 1.6$ vs. $7.1 \pm 1.7$, pre- and postintervention, respectively, $p<0.02$ ), but not rest pain. $n$ (Table 4). Intervention did not have an effect on quality of life (15-D).

\subsection{Responses to visual sensorimotor actions}

After the intervention, patients reported significantly less baseline rest pain at the beginning of the video experiment compared with that before the intervention. The difference remained also during action execution and observation. Unpleasantness decreased and force estimations 
diminished closer to normal in post-intervention compared to pre-intervention (Fig. 5).

\section{Discussion}

An integrated 12-week interdisciplinary rehabilitation program decreased CRPS symptom count and a summary of several secondary outcomes showed improvement. The primary outcome measure - the number of self-reported symptoms - is partly similar to the CRPS severity score that was published and validated after we had started our study [22, 31]; the severity score has been proposed for clinical monitoring and outcome measurement in CRPS research. Our results support the utility of CRPS symptom scoring: during our intervention; the symptom count reduced approximately 15\%, and improvement was detected in patients' motor functioning. Movement pain intensity decreased significantly (11\%), but not rest pain intensity. In general, it is common in CRPS interdisciplinary studies not to find much change in pain intensity, but rather in function $[6,7]$.
A

Execution

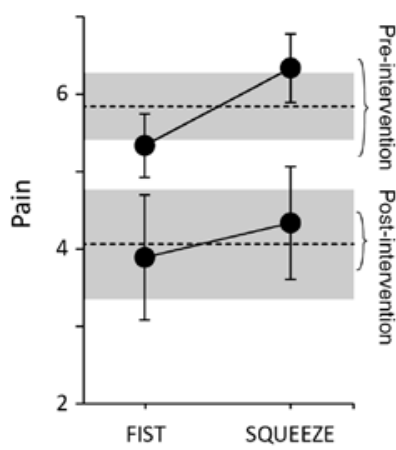

- Affected hand

O Healthy hand

.... Pre-experiment pain
B

Pre-intervention
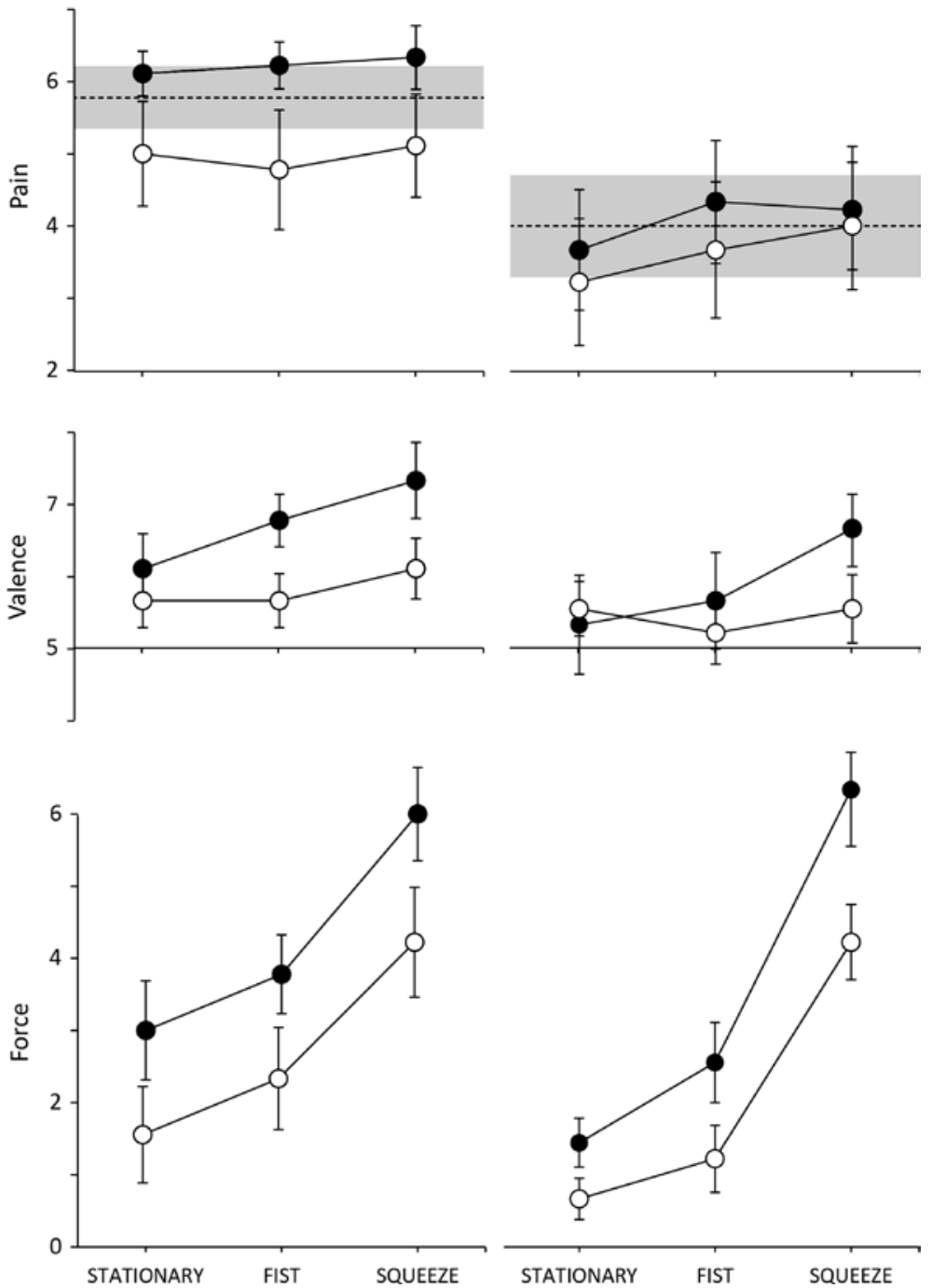

Fig. 5: Responses to visual sensorimotor actions. Pre- and post-intervention NRS-11 ratings (mean \pm SEM) for (A) hand action, and (B) Hand action observation. Pain: in post-intervention, patients began the experiment with lesser rest pain (pre-experiment pain: $p<0.05$, Wilcoxon signed-rank test). The difference was apparent also during action execution $(p<0.05$, ANOVA) and observation $(p<0.05$, ANOVA). Valence: The action observation was less unpleasantness after the intervention $(p<0.05$, ANOVA). Force: ratings of applied force for STATIONARY and FIST videos were decreased, i.e. closer to normal ( $p<0.05$ and $p<0.01$, respectively, ANOVA) [14]. 
Interdisciplinary interventions are necessary in the rehabilitation of chronic CRPS patients. Because pain intensity rarely decreases substantially or significantly, other additional outcomes are necessary to study intervention efficacy $[6,7]$. One previous prospective study assessed the effectiveness of a 4-week interdisciplinary, multimodal pain management program for 12 CRPS type 1 patients using functional measurements: all participants underwent 20 sessions of physiotherapy, 20 sessions of occupational therapy, 12 sessions of water therapy, normal standard medical treatment, stellate ganglion blocks, and 20 sessions of group psychotherapy. Results showed significant improvements in upper extremity motor functioning, while decrease in pain intensity was not significant. There was likewise no significant change in psychological variables, but the patient rating for benefit was positive [6]. Our results with improved motor functioning, but no significant changes in psychological variables or rest pain intensity are in line with these.

Another recent study, a 4-week outpatient interdisciplinary pain management program, showed improvements in physical and emotional functioning in 49 CRPS patients [7]; again, pain intensity did not decrease significantly. The study included CBT, mindfulness-based interventions, relaxation training, occupational therapy, and medical management. There were changes towards more adaptive coping with pain and acceptance of pain. We did not find a significant change towards acceptance of pain as measured by the CPAQ, but patients reported better coping with pain after the intervention, which helped them to accept their condition.

Recently, pain exposure physical therapy has been shown to be similar or superior to conventional treatments in CRPS patients [32, 33]. Exposure in vivo aims to reduce pain-related fear of movement and thereby to reduce painrelated disability. Exposure to daily activities may lead to better results than the usual pain-contingent treatment [33]. In our study, therapy exposed patients to pain as in conventional therapies but led them to face the pain more often with guided home exercises providing repeated exposure. Graded exposure and behavioral activation were conducted in physiotherapy and psychological intervention in a synchronized manner, which encouraged the patients to practice new skills to enable better functioning. The patients also first applied mental imagery exposure in various actions before exposed themselves to similar reallife situations. This integrated intervention aiming for the same goal was acceptable to patients. Exposure therapy shows interesting new avenues for CRPS therapy with new emphasis both from physiotherapy and psychological intervention.

There is growing interest in exploring the effects of pain exposure in a virtual-reality setting. The method could be especially beneficial with patients that have difficulty with imaginal exposure. One study explored the effect of virtual-reality exposure therapy on pain catastrophizing in fibromyalgia patients. Functional magnetic resonance imaging showed that exposing patients to visual exercises elicited changes in brain areas associated with pain catastrophizing [34].

Another recent study applied virtual reality exercises for CRPS type 1 patients [35]. There, the treatment group watched a video clip presenting virtual body swapping. They were asked to assume a posture similar to the body on the screen and to mentally rehearse the movements. The treatment group showed improvement in body perception while the control group did not, despite no group difference in pain levels. Virtual reality exercises may provide a useful tool for CRPS rehabilitation in the future, given frequent body perception disturbances and other wide symptomology in CRPS.

As psychological methods, our study used acceptance and exposure strategies with mindfulness. Exposure and gradual increase of activity were bound to personal goals and values. ACT could be an alternative to CBT in conditions where certain feelings, thoughts and avoidance of physical sensations may increase pain [36, 37]. In our study, a variety of ACT methods were used alongside CBT, aiming to improve acceptance and psychological flexibility [38, 39]. Integrated therapy with ACT and CBT was intended to make it easier to find exercises that suited patients' personal needs, and perhaps this was more effective for function than for distress, the usual target of ACT and CBT. It is unclear why CBT, ACT and exposure did not have the expected effects, apart from in patients' positive accounts: perhaps the therapy was insufficiently intensive; perhaps patients actually practiced at home rather less than they reported, and that practice did not effectively replicate methods taught in face to face contact; or perhaps they wanted to please the therapist by positive feedback. The patients had low levels of anxiety and depressive symptoms at baseline and as the sample size was also small, no statistically significant difference was seen. This may explain why these psychological treatments for chronic pain produced only little change when assessed by questionnaires [40]. In a previous study, CRPS patients have shown a special cognitive deficit with impaired emotional decision-making [41]. This may have played a role in the present study as well with unchanged 
distress variables although reporting positive accounts to the therapist.

The present interdisciplinary treatment was preceded by medication with memantine and morphine as an attempt to reduce pain and help the patients manage exposure exercises better. We conducted the medication intervention according to a previous double-blind placebo-controlled study on memantine-morphine medication and physiotherapy for CRPS patients [15]. That study showed promising effects with decreased pain and neuroimaging findings with decreased activation in pain related areas. Our patients, however, experienced a variety of adverse events that made many of them reduce dosages or discontinue the medication. Serotonergic effects may increase when opioid is combined with serotonergic drugs, which may explain part of their symptoms. All in all, it is not clear why the study medication was not optimal for our patients, although it was similar to the previous trial.

Central nervous system changes are involved in chronic CRPS [42, 43]. We assessed this with action observation known to be unpleasant and even painful for the patients [16] and found these sensations to be milder after the intervention. Our findings and interpretations can be summarized as follows. First, in post-intervention, patients started the experiment with lower baseline pain, which could have been caused e.g. by the familiarity of the setting, and as a consequence, the pain remained at reduced levels throughout the experiment. Second, the intervention did not markedly ameliorate the association between motor actions and pain, as we found no difference between pre- and post-intervention in how action execution or observation modulated pain intensity. Third, and most importantly, the reduced pain levels in post-intervention did not explain the reduction in unpleasantness and force ratings, suggesting these positive effects to be a consequence of the intervention itself to neural plasticity. Less unpleasant viewing of motor actions could enable increased visual attention to the affected limb, better visuomotor control, less effortful actions, and consequently better motor performance.

The study has some limitations. First, the study was underpowered to detect change in many variables. CRPS is a rare disorder and eligible patients were not easy to recruit. Second, the high and unexpected rate of adverse effects from medication may have undermined possible other positive outcomes. Third, there was no follow-up after study completion, so long-term effects of the intervention are not known.

A strength of the study was a careful patient selection and a thorough examination with multiple outcome measures. The motivation of the patients to attend was good with no dropouts and they performed home exercises.
Thus, the integrated therapy model seemed to work well from a patient perspective. Additionally, we performed visual sensorimotor action tests before and after the intervention to demonstrate possible change in neuroplasticity.

\section{Conclusion}

This study supports the previous findings and clinical recommendations of the importance of interdisciplinary treatment for CRPS patients and encourages consideration of an intensive rehabilitation period for chronic CRPS patients in the clinic with an integrated therapy model and an emphasis on exposure in vivo and on reducing distress. We used a novel, broad summary index to pursue observations of change in CRPS features. As a primary outcome, CRPS symptom count showed decrease and for secondary outcomes, motor, pain, and general outcome improved some.

\section{Implications}

The overall summary effect in CRPS symptom load was toward fewer symptoms, less pain, and better motor functioning after the intervention. These results call for an interdisciplinary, integrated therapy for CRPS patients, even those with chronic symptomatology, to improve outcome.

Acknowledgements: We are grateful to physiotherapists Marika Hillo and Leena Honkimaa for conducting the motor assessments and research nurses Soile Haakana and Leena Murto for their excellent contribution. We also thank Professor Emeritus Vesa Niskanen for statistical advice.

\section{Authors' statements}

Research funding: The study was funded by a Governmental Research Grant (TYH2016222, EK) and SHOK, SalWe Ltd., Finland (NF).

Conflict of interest: The authors have no conflicts of interest regarding this paper.

Informed consent: All patients gave their written informed consent.

Ethical approval: The Ethical board of the Helsinki University Hospital and Uusimaa district approved the study (310/13/03/01/2010) and the Finnish Medicines Agency (FIMEA) gave permission for the medical intervention (EudraCT number: 2010-024558-12). The trial was registered in ClinicalTrials.gov (NCT02467556).

Author contributions: Study design: ME, JH, EK, HH, AÄ. Patient selection and clinical examination: HH. Statistical 
analysis: ME, JH. Writing of the article and scientific contribution to it: ME, JH, EK, HH, AW, AÄ, NF.

\section{References}

[1] Harden RN, Bruehl S, Perez RS, Birklein F, Marinus J, Maihofner C, Lubenow T, Buvanendran A, Mackey S, Graciosa J, Mogilevski M, Ramsden C, Chont M, Vatine J-J. Validation of proposed diagnostic criteria (the "Budapest Criteria") for complex regional pain syndrome. Pain 2010;150:268-74.

[2] Bean DJ, Johnson MH, Heiss-Dunlop W, Kydd RR. Extent of recovery in the first 12 months of complex regional pain syndrome type-1: a prospective study. Eur J Pain 2016;20:884-94.

[3] Bean DJ, Johnson MH, Kydd RR. The outcome of complex regional pain syndrome type 1: a systematic review. J Pain 2014;15:677-90.

[4] Schwartzman RJ, Erwin KL, Alexander GM. The natural history of complex regional pain syndrome. Clin J Pain 2009;25:273-80.

[5] Bruehl S, Chung OY. Psychological and behavioral aspects of complex regional pain syndrome management. Clin J Pain 2006;22:430-7.

[6] Singh G, Willen SN, Boswell MV, Janata JW, Chelimsky TC. The value of interdisciplinary pain management in complex regional pain syndrome type I: a prospective outcome study. Pain Physician 2004;7:203-9.

[7] McCormick ZL, Gagnon CM, Caldwell M, Patel J, Kornfeld S, Atchison J, Stanos S, Harden RN, Calisoff R. Short-term functional, emotional, and pain outcomes of patients with complex regional pain syndrome treated in a comprehensive interdisciplinary pain management program. Pain Med 2015;16:2357-67.

[8] de Boer RD, Marinus J, van Hilten JJ, Huygen FJ, van Eijs F, van Kleef M, Bauer MC, van Gestel M, Zuurmond WW, Perez RS. Distribution of signs and symptoms of complex regional pain syndrome type I in patients meeting the diagnostic criteria of the International Association for the Study of Pain. Eur J Pain 2011;15:830.e1-8.

[9] Stanton-Hicks M, Baron R, Boas R, Gordh T, Harden N, Hendler $\mathrm{N}$, Koltzenburg M, Raj P, Wilder R. Complex regional pain syndromes: guidelines for therapy. Clin J Pain 1998;14:155-66.

[10] Punt TD, Cooper L, Hey M, Johnson MI. Neglect-like symptoms in complex regional pain syndrome: learned nonuse by another name? Pain 2013;154:200-3.

[11] Moseley GL. Graded motor imagery is effective for long-standing Complex Regional Pain Syndrome: a randomized controlled trial. Pain 2004;108:192-8.

[12] Smart KM, Benedict MW, O'Connell NE. Physiotherpy for pain and disability in adults with complex regional pain syndrome (CRPS) types I and II. Cochrane Database Syst Rev 2016;2:CD010853.

[13] Harden RN, Oaklander AL, Burton AW, Perez RS, Richardson K, Swan M, Barthel J, Costa B, Graciosa JR, Bruehl S. Complex regional pain syndrome: practical diagnostic and treatment guidelines, 4th ed. Pain Med 2013;14:180-229.

[14] Hayes SC, Storsahl KD, Wilson KG, editors. Acceptance and commitment therapy the process and practice of mindful change. 2nd ed. New York, NY: Guilford Press, 2011.

[15] Gustin SM, Schwarz A, Birbaumer N, Sines N, Schmidt AC, Veit R, Larbig W, Flor H, Lotze M. NMDA-receptor antagonist and morphine decrease CRPS-pain and cerebral pain representation. Pain 2010;151:69-76.

[16] Hotta J, Harno H, Nummenmaa L, Kalso E, Hari R, Forss N. Patients with complex regional pain syndrome overestimate applied force in observed hand actions. Eur J Pain 2015;19:1372-81.

[17] Zhou G, Hotta J, Lehtinen MK, Forss N, Hari R. Enlargement of choroid plexus in complex regional pain syndrome. Sci Rep 2015;5:14329.

[18] Hotta J, Saari J, Koskinen M, Hlushchuk Y, Forss N, Hari R. Abnormal brain responses to action observation in complex regional pain syndrome. J Pain 2016;18:255-65.

[19] Hotta J, Zhou G, Harno H, Forss N, Hari R. Complex regional pain syndrome: the matter of white matter? Brain Behav 2017;7:e00647.

[20] Moseley GL. Graded motor imagery for pathologic pain: a randomized controlled trial. Neurology 2006;67:2129-34.

[21] Gilpin HR, Keyes A, Stahl DR, Greig R, McCracken LM. Predictors of treatment outcome in contextual cognitive and behavioral therapies for chronic pain: a systematic review. J Pain 2017;18:1153-64.

[22] Harden RN, Bruehl S, Perez RS, Birklein F, Marinus J, Maihofner C, Lubenow T, Buvanendran A, Mackey S, Graciosa J, Mogilevski M, Ramsden C, Schlereth T, Chont M, Vatine J-J. Development of a severity score for CRPS. Pain 2010;151:870-6.

[23] Oxford Grice K, Vogel KA, Le V, Mitchell A, Muniz S, Vollmer MA. Adult norms for a commercially available Nine Hole Peg Test for finger dexterity. Am J Occup Ther 2003;57:570-3.

[24] Hudak PL, Amadio PC, Bombardier C. Development of an upper extremity outcome measure: the DASH (disabilities of the arm, shoulder and hand) [corrected]. The Upper Extremity Collaborative Group (UECG). Am J Ind Med 1996;29:602-8.

[25] Beck A, Steer R, Brown G, editors. The beck depression inventory. Second edition manual ed. San Antonio, TX: The Psychological Corporation, 1996.

[26] Ojala T, Piirainen A, Sipila K, Suutama T, Hakkinen A. Reliability and validity study of the Finnish version of the Chronic Pain Acceptance Questionnaire (CPAQ). Disabil Rehabil 2013;35:306-14.

[27] McCracken LM, Dhingra L. A short version of the Pain Anxiety Symptoms Scale (PASS-20): preliminary development and validity. Pain Res Manag 2002;7:45-50.

[28] Sintonen H. An approach to measuring and valuing health states. Soc Sci Med Med Econ 1981;15:55-65.

[29] Vartiainen P, Mantyselkä P, Heiskanen T, Hagelberg N, Mustola S, Forsell H, Kautiainen H, Kalso E. Validation of EQ-5D and 15D in the assessment of health-related quality of life in chronic pain. Pain 2017;158:1577-85.

[30] Hunsaker FG, Cioffi DA, Amadio PC, Wright JG, Caughlin B. The American Academy of orthopaedic surgeons outcome instruments: normative values from the general population. J Bone Joint Surg Am 2002;84A:208-15.

[31] Harden RN, Maihofner C, Abousaad E, Vatine J-J, Kirsling A, Perez RS, Kuroda M, Brunner F, Stanton-Hicks M, Marinus J, van Hilten J, Mackey S, Birklein F, Schlereth T, Mailis-Gagnon A, Graciosa J, Connoly SB, Dayanim D, Massey M, Frank H, et al. A prospective, multisite, international validation of the Complex Regional Pain Syndrome Severity Score. Pain 2017;158:1430-6.

[32] Barnhoorn KJ, van de Meent H, van Dongen RT, Klomp FP, Groenewoud H, Samwel H, Nijhuis-van der Sanden MW, Frölke JP, Staal JB. Pain exposure physical therapy (PEPT) 
compared to conventional treatment in complex regional pain syndrome type 1: a randomised controlled trial. BMJ Open 2015;5:e008283-2015-008283.

[33] den Hollander M, Goossens M, de Jong J, Ruijgrok J, Oosterhof J, Onghena P, Smeets R, Vlaeyen JWS. Expose or protect? A randomized controlled trial of exposure in vivo vs. pain-contingent treatment as usual in patients with complex regional pain syndrome type 1. Pain 2016;157:2318-29.

[34] Morris LD, Louw QA, Grimmer KA, Meintjes E. Targeting pain catastrophization in patients with fibromyalgia using virtual reality exposure therapy: a proof-of-concept study. J Phys Ther Sci 2015;27:3461-7.

[35] Jeon B, Cho S, Lee JH. Application of virtual body swapping to patients with complex regional pain syndrome: a pilot study. Cyberpsychol Behav Soc Netw 2014;17:366-70.

[36] McCracken LM, Velleman SC. Psychological flexibility in adults with chronic pain: a study of acceptance, mindfulness, and values-based action in primary care. Pain 2010;148:141-7.

[37] Crombez G, Viane I, Eccleston C, Devulder J, Goubert L. Attention to pain and fear of pain in patients with chronic pain. J Behav Med 2013;36:371-8.
[38] Veehof MM, Trompetter HR, Bohlmeijer ET, Schreurs KM. Acceptance- and mindfulness-based interventions for the treatment of chronic pain: a meta-analytic review. Cogn Behav Ther 2016;45:5-31.

[39] Viane I, Crombez G, Eccleston C, Devulder J, De Corte W. Acceptance of the unpleasant reality of chronic pain: effects upon attention to pain and engagement with daily activities. Pain 2004;112:282-8.

[40] Williams ACDC, Eccleston C, Morley S. Psychological therapies for the management of chronic pain (excluding headache) in adults. Cochrane Database Syst Rev 2012;11:CD007407.

[41] Apkarian V, Sosa Y, Krauss B, Thomas S, Fredrickson B, Levy R, Harden N, Chialvo D. Chronic pain patients are impaired in emotional decision-making task. Pain 2004;108:129-36.

[42] Flor H, Diers M. Sensorimotor training and cortical reorganization. NeuroRehabilitation 2009;25:19-27.

[43] Schwenkreis P, Maier C, Tegenthoff M. Functional imaging of central nervous system involvement in complex regional pain syndrome. Am J Neuroradiol 2009;30:1279-84. 